\title{
Prevalence of Staphylococcus Aureus among Children Diagonosed with Acute Diarrhea in Kano, Nigeria
}

\author{
Muhammad Ali*1, Muhammad S Abdallah² and Auwal Umar ${ }^{3}$ \\ ${ }^{1}$ Department of Microbiology, Federal University Gusau, Nigeria \\ ${ }^{2}$ Desert Research Monitoring and Control Centre, Yobe State University Damaturu, Nigeria \\ ${ }^{3}$ Biological Science Unit, Ahmadu Bello University School of Basic and Remedial Studies Funtua, Nigeria \\ *Corresponding author: Muhammad Ali, Department of Microbiology, Federal University Gusau, Nigeria
}

Received: 㯺 March 08, 2019

Published: March 20, 2019

\begin{abstract}
The burden of diarrheal disease is most critical in developing countries, facilitated by unsafe water supplies, poor sanitation, and nutritional deficiencies. The research was aimed to study the prevalence of Staphylococcus aureus among children diagnosed with acute diarrhea in Kura General Hospital Kano, Nigeria. Fecal specimens were collected in clean, dry and leak proof sterile bottle from 58 child patients (ranges from 1-5 years) admitted to Kura General Hospital and diagnosed with acute diarrhea from period of March to August 2017. The isolates were isolated and identified using Gram staining, Biochemical test (Catalase, Coagulase and DNase test), Mannitol fermentation and haemolysis test. The result showed that 34 samples out of 58 were positive for S. aureus. Higher incidence was found among males (20 subjects which accounted for 59\%) than female with total of 14 subjects accounted for $41 \%$. Highest frequency of diarrhea infection is found among subject with age between 1-2 years and more male (53\%) were infected than female (47\%). Statistical analysis of the result showed that there is no considerable statistical difference on prevalence of $\mathrm{S}$. aureus among sex group and age categories of the subject at $\mathrm{p}<0.05$. It is recommended that proper environmental sanitation, good personal hygiene and complete immunization against diarrhea disease are recommended.
\end{abstract}

Keywords: Acute diarrhea; children; prevalence; Staphylococcus aureus

\section{Introduction}

Over 1.7 billion cases of diarrheal infection are reported every year and these are associated with about 2.2 million deaths annually [1]. The burden of the disease is most severe and critical in a developing country which is facilitated by poor sanitation, unsafe water supply and nutritional deficiencies. Diarrhea may be infectious or non-infectious. It can be infectious when caused by bacteria, virus or parasite. Bacteria are one of the major causative agents of food illness such as diarrhea and accounted for about $60 \%$ of cases requiring hospitalization [2]. The global impact of foodborne illness is difficult to assess. However, it has been estimated that about 2.1 million children die due to diarrhea related in developing countries annually. It has been reported that water and food are the vehicle of diarrhea related illness [1]. Due to biological nature of food, it is capable of supplying consumer with nutrients, and hence, equally capable of supporting the growth of contaminating organisms. Three types of bacterial foodborne diseases are recognized: intoxications, infections, and toxico-infections. The foodborne bacterial intoxication is caused as result of ingesting food containing bacterial toxins and such toxins are produced by organisms such as Staphylococcus aureus and Clostridium botulinum, resulting from bacterial growth in the food. According to Infectious Diseases Society of America (IDSA) and the American College of Gastroenterology (ACG), Diarrhea is defined as the passage of three or more loose or liquid stools per day. The diarrhea can be further classified by the duration of the symptoms [3]. Patients diagnosed with acute diarrhea shows symptoms lasting less than 14 days. Those showing symptoms for more than 14 days or 1 month are said to have persistent diarrhea. 
Those having diarrhea for more than 30 days are said to have chronic diarrhea.

Staphylococcus aureus is gram positive bacteria, spherical in shape (cocci) mostly occur in singles, tetrads and irregular grape like cluster. It is the only strain that produce enterotoxins that can cause food poisoning. The food handler with lesion or carriage may initiate infection [4,5]. The species are host adapted with most of the known species inhibiting humans and other animals. These species are found in large number near opening of the body surfaces such as the anterior nares, inguinal and perineal areas. There are six types of enterotoxins produced serologically by $\mathrm{S}$. aureus. These include enterotoxin A, B, C1, C2, D and E and they differ in their toxicity. Most food poisoning is caused by enterotoxin A followed by D. These enterotoxins are heat stable, with type B being most heat resistant. Enterotoxin stimulates Central Nervous Systems (CNS) vomiting center and inhibit water and sodium absorption in the small intestine. Staphylococcal enterotoxins, along with the toxic syndrome toxin and others, are classed as bacterial super antigens relative to in vivo antigen recognition in contrast to conventional antigens [6]. Food poisoning by S. aureus is characterized by a short incubation period typically 2-4 hours. The onset is sudden and is characterized by vomiting and diarrhea but no fever. The illness lasts less than 12 hours. In severe cases dehydration, masked pallor and collapse may require treatment (intravenously) infusion. The short incubation periods are the characteristics of intoxication where illness is the results of ingestion of the preformed toxin in the food [7]. The research was aimed to study the prevalence of S. aureus among children diagnosed with acute diarrhea in Kura General Hospital Kano, Nigeria.

\section{Materials and Methods}

\section{Ethical approval}

Ethical approval (with reference no. BHM/GEN/488/VOL.1) for the study was obtained from Kano State Hospital Management Board (HMB) based on the consent of Kura General Hospital ethical committee.

\section{Study area}

The samples for the study were collected at Kura General Hospital in Kura local Government area of Kano State, Nigeria. It is located at a distance of about 35 kilometer south west from the state capital. Kura is located at Latitude $11046^{\prime} 17^{\prime \prime} \mathrm{N}$ and Longitude $8025^{\prime} 49^{\prime \prime}$ E. It covers an area of about $206 \mathrm{Km} 2$ of land and population of about 144,601 according to 2006 census [8]. Kura local government share common boundaries with Dawakinkude (East), Garun-mallam (West), Madobi (North) and Bunkure (South) [8].

\section{Sample Collection}

Fecal specimens were collected in clean, dry and leak proof sterile bottle from 58 child patients (ranges from 1-5 years) admitted to Kura General Hospital and diagnosed with acute diarrhea from period of March to August 2017. The specimens were immediately transported to Microbiology Laboratory in the Department of Microbiology, Kano University of Science and Technology Wudil for isolation and identification of S. aureus.

\section{Isolation of $\mathbf{S}$. aureus}

A sterile wire loop was deep into the fecal sample of the patients and streaked onto the surface of Nutrient agar (Life save Biotech, USA), using standard method described by of Prescott et al. [9]. This procedure was applied for each of the sample and the plates were incubated at $370 \mathrm{C}$ for 24 hours. The presumptive colonies of S. aureus on the plates were further sub-cultured to obtained pure culture. The pure isolates of S. aureus were preserved for further bacterial identification.

\section{Bacterial Identification}

The bacteria isolated were confirmed as S. aureus by conventional microbiological methods namely; Gram staining, biochemical test (such as catalase, coagulase and DNase test), mannitol and haemolysis test. Gram staining was done according to the methods described by Chessbrough [10]. Catalase, Coagulase and DNase test were done according to the method described by Holt et al. [11] and Cheesbrough [10]. Mannitol fermentation and haemolysis test was done according to the method described by Holt et al. [11].

\section{Gram Staining}

Gram staining was done according to method described by Cheesbrough [10]. A thin smear was made by emulsifying an overnight culture of the isolate in normal saline on a well labeled clean glass slide. The smear was air dried and fixed by heat. This is followed by flooding the slide with crystal violet as primary stain for 30 seconds and then rinsed the slide with distilled water. The smear was flooded with Lugol's iodine as a mordant to fix the primary stain and then rinsed with distilled water after 60 seconds. The slide was decolorized using acetone and rinsed immediately. Counter stain with safranin followed and left for 30 before being rinsed off. The stain smear was air dried and observed under microscope.

\section{Biochemical Tests}

\section{Catalase test}

Catalase test was conducted using procedure described by Holt et al. [11]. A drop of $3 \%$ hydrogen peroxide was placed on a clean glass slide. An overnight culture of the isolate was picked using sterile wire loop and placed on the drop of the hydrogen peroxide; presence of bubbles observed indicated a positive catalase test.

\section{Coagulase test}

Three drops of blood plasma were placed on clean and greasefree slide. A colony of the isolate was picked by means of sterile wire 
loop from incubated Nutrient agar plate. The coony was emulsified in the blood plasma and observed for clot formation [11].

\section{DNase test}

Deoxyribonuclease agar medium was prepared according to manufacturer's instructions. An overnight over night broth culture of the isolate was spot inoculated on the surface of the medium and incubated at $370 \mathrm{C}$ for 24 hours. At the end of the incubation period, the agar surface was flooded with $1 \mathrm{~N}$ hydrochloric acid and excess drained off [10].

\section{Bacteriological analysis}

The pure isolate obtained on Nutrient agar medium was picked using sterile wire loop and inoculate on the surface of Mannitol salt agar and blood agar (Lifesave Biotech, USA) and incubated at $370 \mathrm{C}$ for 24 hours. The changes in color of the medium from pink to yellow indicated positive results [11].

\section{Statistical analysis}

The prevalence of S. aureus isolates between sex and age categories was compared by using the Chi-square test (SPSS Version 19). Differences between the prevalence rates were considered significant when $\mathrm{p}<0.05$.

\section{Results}

\section{Demographic distribution of patients}

The demographic distribution of child patients diagnosed with acute diarrhea is presented in (Table 1). A total of 58 subjects (31 male and 27 female) were considered in the study. The age distribution of the subjects ranged from 1-5 years. Highest frequency is found among subject with age between 1-2 years.

Table 1: Demographic distribution of child patients diagnosed with acute diarrhea

\begin{tabular}{|ccc|}
\hline Parameters & Frequency (n) & Percentage (\%) \\
\hline Age (year) & & \\
Less than 1 & 11 & 19 \\
$1-2$ & 15 & 26 \\
$2-3$ & 14 & 24 \\
$3-4$ & 10 & 17 \\
$4-5$ & 8 & 14 \\
Sex & & \\
Male & 31 & 53 \\
Female & 27 & 47 \\
\hline
\end{tabular}

\section{Identification of S. aureus}

The identification of S. aureus from the fecal samples of the subjects is presented in the table below (Table 2). The S. aureus was identified using Gram staining, biochemical test and Mannitol fermentation test. Result showed that the isolates were positive for Gram staining, catalase, coagulase, DNase and Mannitol fermentation test. The isolates showed $\beta$-haemolysis on blood agar plates.
Table 2: Morphological and Biochemical Identification for $S$. aureus.

\begin{tabular}{|ccc|}
\hline S/N & Test & Inference \\
\hline 1 & Catalase & + \\
2 & Coagulase & + \\
3 & DNase & + \\
4 & Mannitol test & Golden yellow colony \\
5 & Blood agar growth & $\beta$-haemolysis \\
6 & Nutrient agar growth & Yellow colony \\
\hline
\end{tabular}

\section{Prevalence of S. aureus}

The prevalence of S. aureus among fecal samples of the subjects is presented in (Table 3) below. A total of 58 fecal samples were examined for the presence of $\mathrm{S}$. aureus. The result showed that 34 samples were positive for $\mathrm{S}$. aureus. Higher incidence was found among males (20 subjects which accounted for $59 \%$ ) than female with total of 14 subjects accounted for $41 \%$. The prevalence of S. aureus among fecal samples of the subjects in relation to age category is presented in (Table 4). A total of 58 fecal samples were examined for the presence of S. aureus. The result showed that 34 samples were positive for S. aureus. Higher incidence was found among subjects with age category $2-3$ with prevalence of $17.2 \%$. Least prevalence was recoding among subjects with age category less than 1 with $6.6 \%$.

Table 3: Prevalence of $S$. aureus with respect to sex.

\begin{tabular}{|ccccc|}
\hline Sex & $\begin{array}{c}\text { Samples } \\
\text { examine }\end{array}$ & $\begin{array}{c}\text { Staph } \\
\text { positive (n) }\end{array}$ & $\begin{array}{c}\text { Prevalence } \\
\text { (\%) }\end{array}$ & $\mathbf{X}^{\mathbf{2}}$ \\
\hline Male & 31 & 20 & 34 & $0.2507^{*}$ \\
Female & 27 & 14 & 24 & \\
Total & 58 & 34 & 58 & \\
\hline
\end{tabular}

Key: *The $p$-value is 0.616589 . The result is statistically not significant at $p<0.05$

Table 4: Prevalence of $S$. aureus with respect to age of the subject.

\begin{tabular}{|ccccc|}
\hline Age (years) & $\begin{array}{c}\text { Samples } \\
\text { examine } \\
\text { (n) }\end{array}$ & $\begin{array}{c}\text { Staph } \\
\text { positive (n) }\end{array}$ & $\begin{array}{c}\text { Prevalence } \\
\text { (\%) }\end{array}$ & $\mathbf{X}^{2}$ \\
\hline Less than 1 & 11 & 4 & 6.6 & $3.1776^{*}$ \\
$1-2$ & 15 & 5 & 8.6 & \\
$2-3$ & 14 & 10 & 17.2 & \\
$3-4$ & 10 & 8 & 13.6 & \\
$4-5$ & 8 & 7 & 12 & \\
Total & 58 & 34 & 58 \\
\hline
\end{tabular}

Key: *The p-value is 0.528557 . The result is statistically not significant at $\mathrm{p}<0.05$

\section{Discussion}

Acute diarrhea remains as one of the most prevalent diseases affecting children in developing countries. The associated morbidity and mortality of the disease in children under five years of age is a major health problem in those countries. The present study 
was aimed at determining the prevalence of S. aureus in the fecal sample child patients diagnosed with acute diarrhea attending Kura General Hospital Kano. The finding of the study indicated that 34 out of the 58 samples were positive for S. aureus which accounted for $58 \%$ of the sample collected. Higher incidence was found among subjects with age category 2- 3 years with prevalence of $17.2 \%$. Least prevalence was recoding among subjects with age category less than 1 with $6.6 \%$. Higher incidence of S. aureus among subject with age category 2-3 years is due to increased susceptibility to microbial infection, and this can be explained by the decline in the passive immunity because they no longer take breast feeding from their mothers due to weaning and this increase exposure to the diarrheal agents. Identification of S. aureus in the present study was based on Gram staining, cultural characteristics and biochemical characterization. All the 34 isolates were able to ferment Mannitol producing yellow colony, they also showed $\beta$-haemolysis on blood agar medium enriched with $5 \%$ sheep blood. Gram staining of the isolates exhibited a cluster of Gram-positive cocci. The isolates were positive for catalase, coagulase and DNase test. In catalase test, hydrogen peroxide was broken down into water and oxygen by enzyme catalase. The production of oxygen was indiated by bubble formation [12]. The positive result of coagulase test was confirmed by the formation of curd like clotting compared to negative control [13]. Earlier findings by Amengialue, et al. [13], Yabaya, et al. [14], Jahan, et al. [12] identified and characterized S. aureus on the basis of cultural characteristics, Gram staining and Biochemical characterization. The presence of S. aureus in the fecal samples of the subjects in the present study demonstrated that the isolate is one of the causative organisms associated with acute diarrhea in children. If food is stored for sometimes in room temperature the organism may in the food and can produce toxin. The bacteria produce enterotoxin while multiplying in food. S. aureus is known to produce six serologically different types of enterotoxins (A, B, C1, C2, D and E) that differ in toxicity [15]. The acute diarrhea is caused by ingestion of food containing viable bacteria which then grow and establish themselves in the host and subsequently produce a toxin in the host. In the present study, a total of 58 cases of acute diarrhea were recorded with male having higher incidence of the cases $(53 \%)$ than female child (47\%). This suggests that male suffer more frequently from diarrhea compared to female in the study area. This finding correlate with that of Uche et al. [16] who assess the incidence of diarrhea in children under 5 years at the Institute of Child Health, Banzazzau Zaria, Nigeria and found higher incidence among male child than female with percentage incidence of $55 \%$ and $45 \%$ for male and female respectively. According to the presence study, age was a significant factor in the prevalence of diarrheal disease. Age specific prevalence of diarrhea was higher in the 1 - 2 years age group. This correlate with the studies conducted by El-Alamy, et al. [16] and Biritwum, et al. [17] who studied incidence of diarrheal disease in Egypt and Ghana respectively. The prevalence of diarrhea based on age category in this study was in conformity with that of [18] who found the highest prevalence of diarrhea by age group among children was 12-23 months (36.8\%) while older children in the group $<48$ months of age had the least prevalence $(6.4 \%)$. The increased susceptibility of this age group to the diarrheal disease can be explained by the decline in the passive immunity and increased exposure to the diarrheal agents at this age. There is agreement that children less than 2 years of age are most affected by diarrhea [19] while some researchers have identified an increase incidence of the disease in children younger than one year [19]. It has been clear that weaning take place at this age category (1-2 years) which exposes the child to environmental contaminants in poor hygienic condition. Thus, early weaning is a putative factor to the onset of diarrhea in this age range. Therefore, early weaning and incomplete immunizations have a synergistic effect in enhancing disease.

\section{Conclusion}

Based on the finding of the study, S. aureus is one of the etiological agents of diarrhea. From the 58 fecal sample of examined, 34 samples which accounted for $58 \%$ were found to be positive for S. aureus. Higher incidence of the isolate was found among subjects with age category $2-3$ with prevalence of $17.2 \%$. High prevalence of $\mathrm{S}$. aureus among the subject was attributed to weaning, poor hygienic condition and lack of immunization. In the study, it is also found that higher incidence of diarrhea is more in male subjects than the female counter parts. It is recommended that proper environmental sanitation and delayed weaning is encouraged. Complete immunization against diarrhea disease is also recommended.

\section{Acknowledgement}

The authors wish to thank Department of Health, Kura Local Government for their support and cooperation. Thanks to staff of Kura General Hospital for sample provision. We also wish to acknowledge the contribution of technical staff of Microbiology Department, KUST Wudil for use of laboratory facilities.

\section{References}

1. (2008) World Health Organization. Food borne disease outbreaks: guide line for integration and control. Geneva.

2. Checkley W, Buckley G, Gilman RH, Assis AM, Guerrant RL, et al. (2008) Multi-country analysis of the effects of diarrhea on childhood stunting. Int J Epidemiol 37: 816-830.

3. Guerrant RL, Van Gilder T, Steiner TS, Thielman NM, Slutsker L, et al. (2001) Practice guidelines for the management of infectious diarrhea. Clin Infect Dis 32: 331-351.

4. Quinn PJ, Markey BK, Carter ME, Demnelly WJ (2001) Veterinary microbiology and microbial disease. (8thedtn) Blackwell publishing, oxford, UK.

5. Walderhaug M (2007) Food borne pathogenic microorganisms and natural toxin Food and Drug Administration. Center for Food Safety and Applied Nutrition 28: 48-65.

6. Anderson K and Pritchard D (2008) An Update on S. aureus Mastitis. (4 $4^{\text {th }}$ edn), Benjamin/ Cummings Publishing Company.

7. Adams M and Moss M (2008) Food microbiology ( $3^{\text {rd }}$ edn), UK RSC press, pp. 252256. 
8. (2006) National Population Commission (NPC). National population census result, Abuja Nigeria.

9. Presscot LM, Harley JP, Klein DA (2005) Microbiology Sixth edition. McGraw Hill International edition New York.

10. Chessbrough M (2010) District laboratory practice in tropical countries, second edition, part two, Cambridge University Press, p. 80-85.

11. Holt JG, Krieg NR, Sneath PA, Stanley JT, Williams ST (1994) Bergey's manual of systematic bacteriology, 9th edition. Williams \& Wilkins Co. Baltimore, Maryland, p. 786.

12. Jahan M, Rahman M, Parvej S, Shah M, Chowdhury ZH et al. (2005) Isolation and characterization of $S$. aureus from raw cow milk in Bangladesh. J Adv Vet Anim Res 2(1): 49-55.

13. Amengialue 00, Osawe FO, Edobor O, Omoigberale MNO, Egharevba AP (2015) Prevalence and antibiogram pattern of $S$. aureus in urinary tract infection among patients attending specialist hospital. Benin City, Nigeria, GJBAS 2(4): 46-49.

14. Yabaya A, Jeremiah MY, Manga SS, Umar A (2011) Prevalence and antimicrobial susceptibility pattern of $S$. aureus isolated from the skin and Nasal cavity of students and staff of Kaduna state University, Kaduna, Nigeria. Best Journal 8(2): 191-194.
15. Centers for Disease Control and Prevention (2011) Vital signs: incidence and trends of infection with pathogens transmitted commonly through food-foodborne diseases active surveillance network, 10 U.S. sites, 1996-2010. MMWR Morb Mortal Wkly Rep 60: 749-755.

16. Ucheh IB, Eleojo AA, Tyoalumun K, Nanpen DM (2017) Assessment of the incidence of diarrhea in children under 5 years at the Institute of Child Health, Banzazzau, Zaria. Ann Nigerian Med 11: 6-10.

17. El Alamy MA, Thacker SB, Arafat RR, et al. (1986) The incidence of diarrheal disease in defined population of rural Egypt. Am J Trop Med Hyg 35(5): 1006-12.

18. Biritwum RB, Isomura S, Assoku A, Torigol S (1986) Growth and diarrhoeal disease surveillance in a rural Ghananian preschool child population. Trans R Soc Trop Med Hyg 80(2): 208-13.

19. Gracey M and Walker Smith A (1997) Diarrheal disease. Nestle Nutrition Workshop Series 38: 1-340.

20. Giugliano LG, Bernardi MG, Vasconcelos JC et al. (1986) Longitudinal study of diarrhoeal disease in a peri-urban community in Manaus (Amazon-Brazil). Ann Trop Med Parasitol 80: 443- 50.
To Submit Your Article Click Here: Submit Article

DOI: 10.32474/MAMS.2019.01.000110

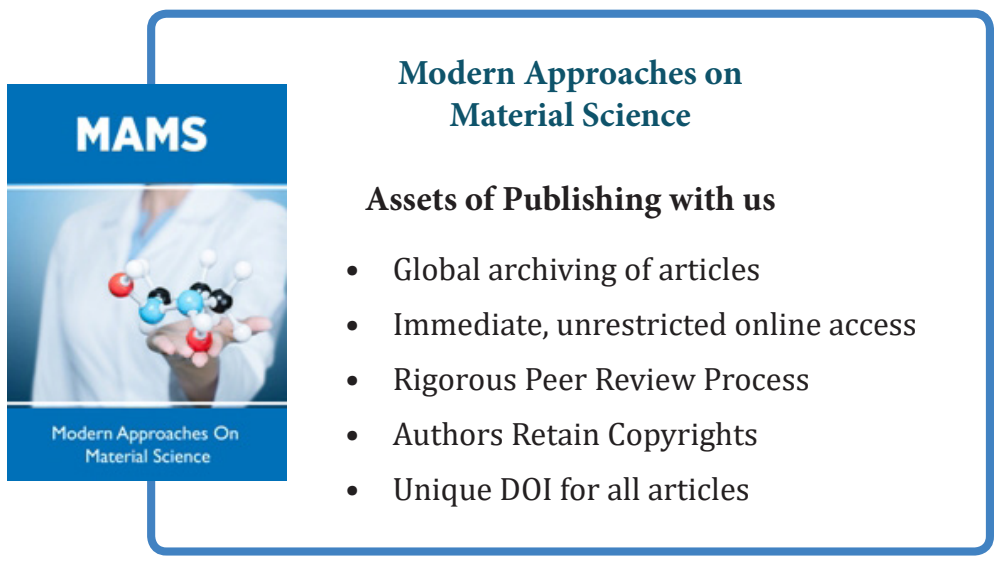

